\title{
Secondary Vertexing in REDTOP Experiment Simulations
}

\author{
K. Maamari ${ }^{1}$, C. Gatto ${ }^{234}$ on behalf of the REDTOP collaboration ${ }^{345}$ \\ ${ }^{1}$ University of Southern California, ${ }^{2}$ Istituto Nazionale di Fisica Nucleare - Sezione di Napoli, ${ }^{3}$ Fermi National Accelerator Laboratory, ${ }^{4}$ Northern Illinois University, ${ }^{5}$ Arizona State \\ University, Benemérita Universidad Autónoma de Puebla, Brown University, Budker Institute of Nuclear Physics - Novosibirsk, IFAE - Barcelona, Istituto Nazionale di Fisica \\ Nucleare - Sezione di Ferrara, Istituto Nazionale di Fisica Nucleare - Sezione di Pisa, Jagiellonian University - Krakow, Los Alamos National Laboratory, National Centre for Nuclear \\ Reggio Emilia, Universita' di Pisa, Università di Salerno, University of Florida, University of Kentucky, University of Minnesota, University of Uppsala

\section{ABSTRACT}

The $\eta / \eta$ ' mesons are almost unique in the particle universe since they are Goldstone bosons and the dynamics of their decays are strongly constrained. Because the $\eta / \eta$ ' have no charge, decays that violate conservation laws can occur without interfering with a corresponding current. The integrated eta meson samples collected in earlier experiments have been less than $\sim 10^{9}$ events, limiting considerably the search for such rare decays. The $\eta$ ' sample is even more scarce. REDTOP (Rare Eta Decays with a TPC for Optical Photons) is being proposed at the proton booster of Fermilab with the intent of collecting more than $10^{15}$ triggers/year for studies of rare $\eta / \eta$ ' decays. Such statistics are sufficient for investigating several symmetry violations, and for searches for new particles beyond the Standard Model. This following project is most concerned with the detection of such new particles by way of the identification of detached, secondary vertices.

\section{— INTRODUCTION TO THE REDTOP EXPERIMENT}

The REDTOP experiment belongs to the High Intensity class of experiments, using a $1.8 \mathrm{GeV}$ continuous proton beam impinging on a target composed by 10 foils of beryllium to produce about $10^{13} \eta$ mesons in one year of running. The detector will attempt to capture the decay products of the $\eta$ mesons, in particular the ones that are either not expected or suppressed at the $10^{-11}$ level. The REDTOP detector (Figure 1) utilizes an Optical TPC and the ADRIANO Dual-Readout Calorimeter in addition to a fiber tracker. The OTPC operates on the same principles as a conventional TPC, however using the Cherenkov effect to detect the particles. The ADRIANO Calorimeter utilizes both scintillation and the Cherenkov processes in two separate optical media to identify particles.

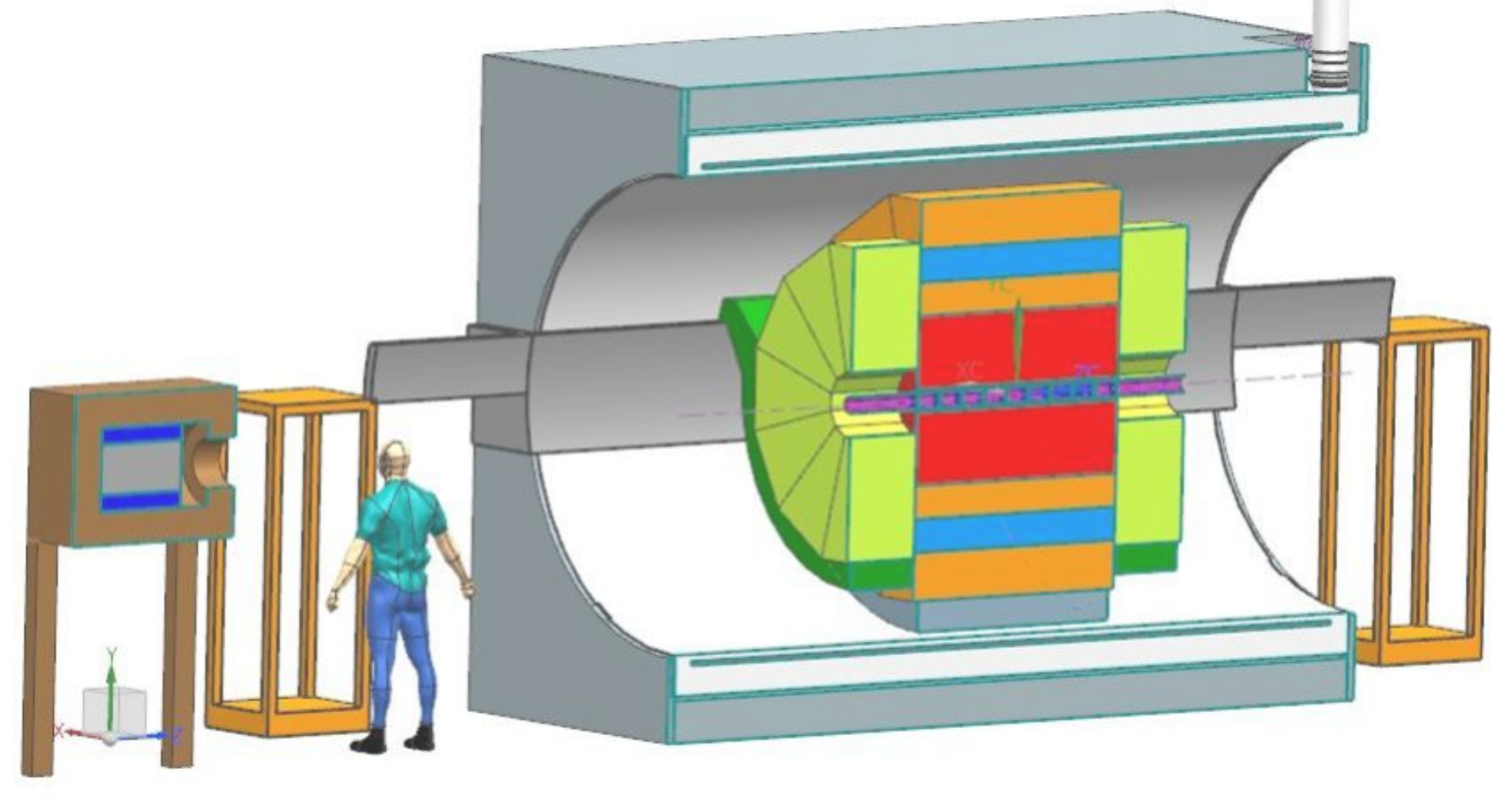

Figure 1: The REDTOP Detector.

\section{SECONDARY VERTEX IDENTIFICATION}

Long lived particles such as the dark Higgs and dark photon travel prior to decaying once more, hence producing a secondary vertex, as pictured in Figure 2. Some common $\eta$ decays producing secondary vertices include:

$$
\begin{aligned}
p+L i \rightarrow \eta+X \text { with } \eta & \rightarrow \gamma+A^{\prime} \text { and } A^{\prime} \rightarrow e^{+}+e^{-} \\
\eta & \rightarrow \pi^{0}+H \text { and } H \rightarrow e^{+}+e^{-}
\end{aligned}
$$

However, $e^{+} e^{-}$combinations may also occur from the initial decay of the $\eta$ such as in the following cases:

$$
\begin{aligned}
p+L i \rightarrow \eta+X \text { with } \eta & \rightarrow \gamma+e^{+}+e^{-} \\
\eta & \rightarrow \pi^{0}+e^{+}+e^{-}
\end{aligned}
$$

Thus, to differentiate primary $e^{+} / e^{-}$tracks from secondary tracks, each track was fit to the position of the beam. The chi-squared value for of the resulting fit was used to classify tracks as either secondary or primary. All primary tracks were, once more, fit to the position of the beam whereas secondary tracks were fit direction of the primary-secondary vertex line, thus creating a primary and secondary vertex for each event. This procedure is highlighted in Figure 3

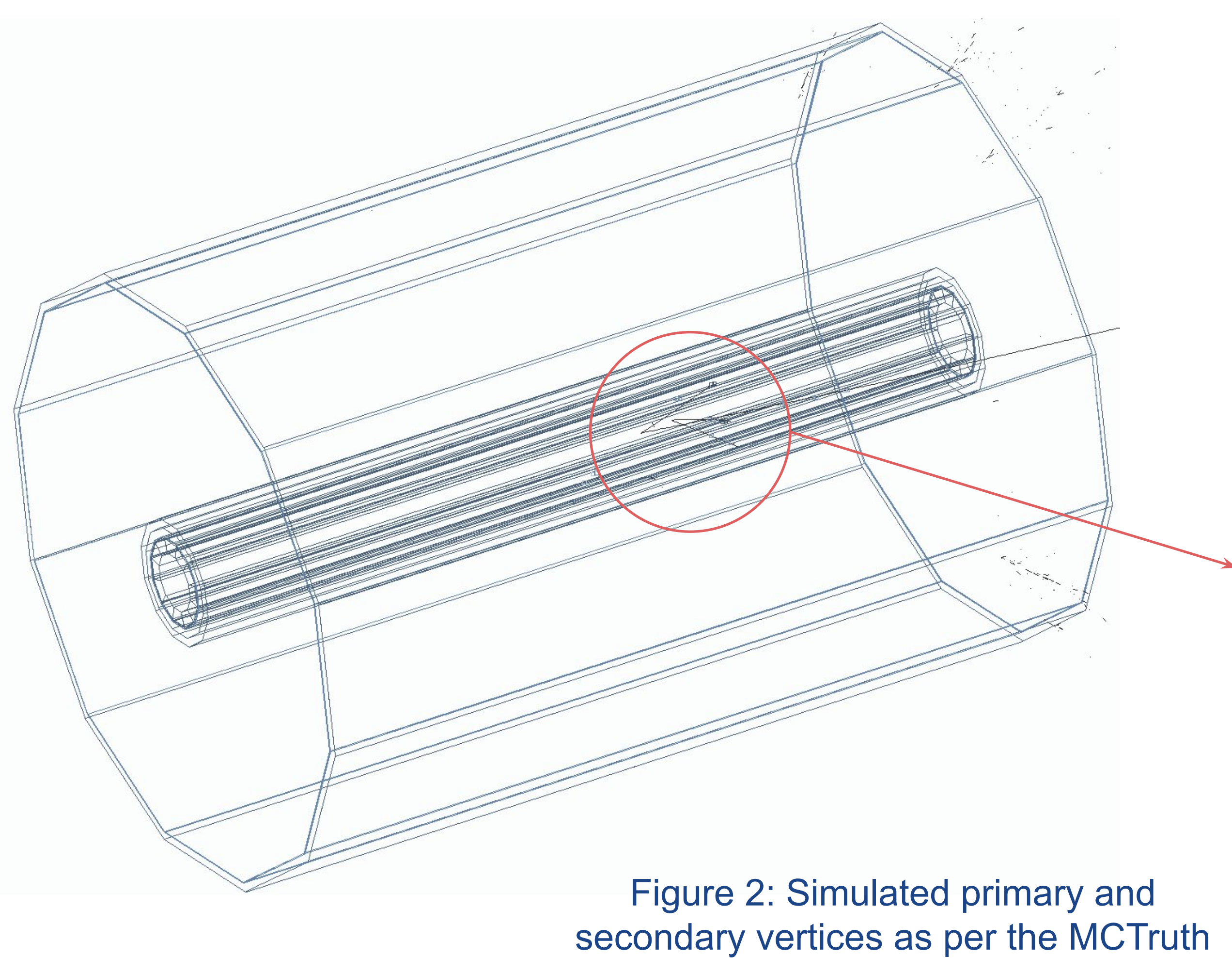

\section{- INVARIANT MASS RECONSTRUCTION}

The identification of secondary vertices allows for the rejection of background, in turn improving the S/B ratio. An example of a hypothetical $400 \mathrm{MeV}$ dark photon is demonstrated below in the reconstruction of the invariant masses of the electrons and positrons (Figures 4, 5, 6, and 7).
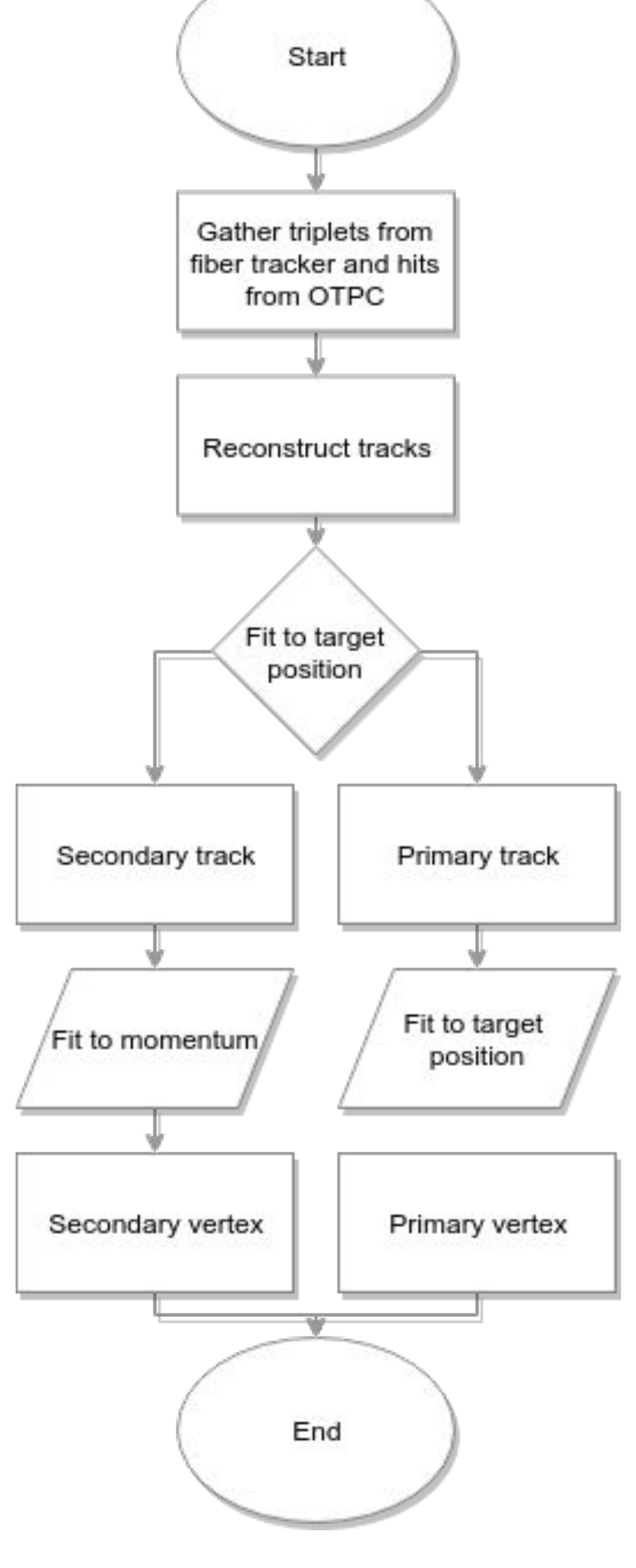

Figure 3: An outline of the procedure taken to determin primary and secondary vertices

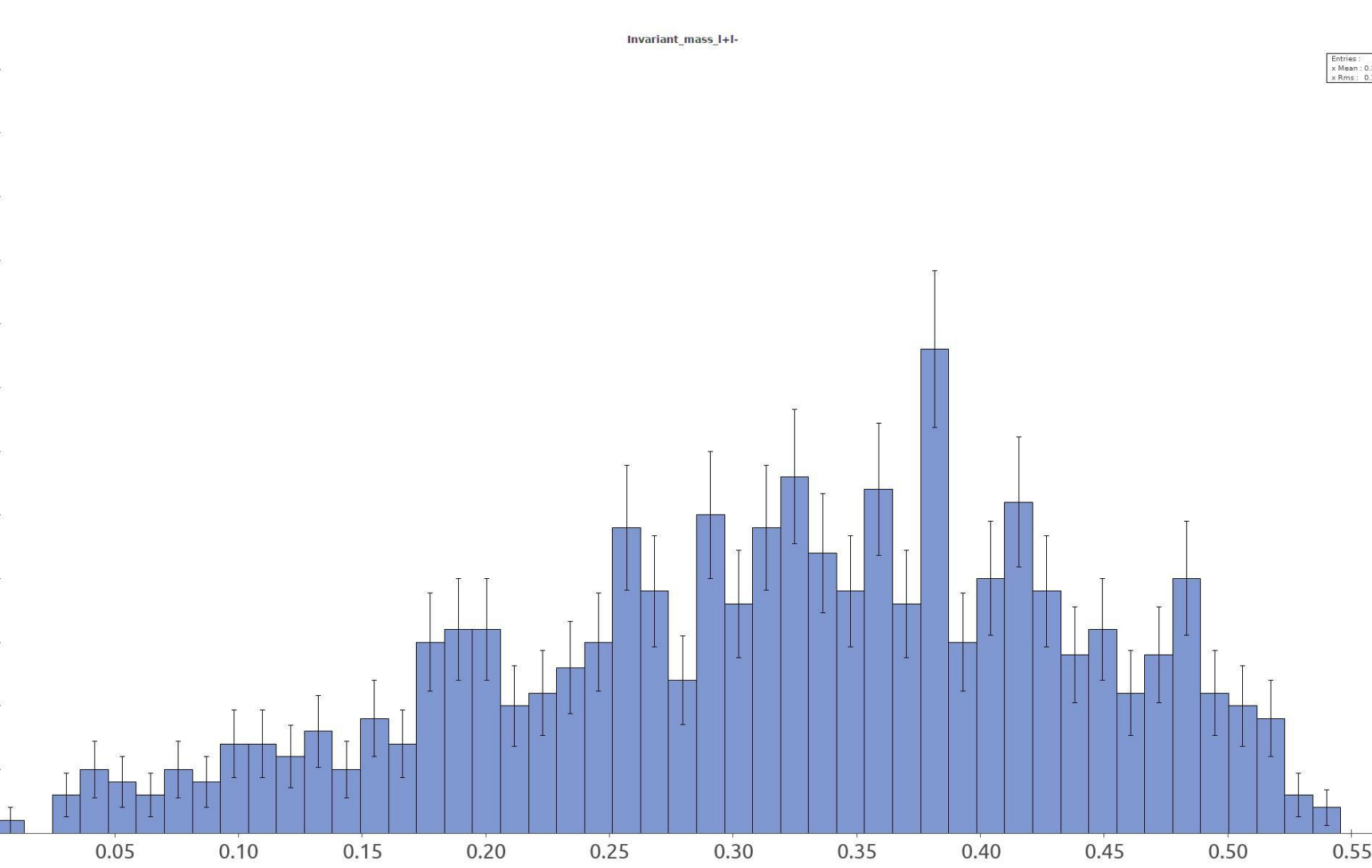

Figure 4: Background detections

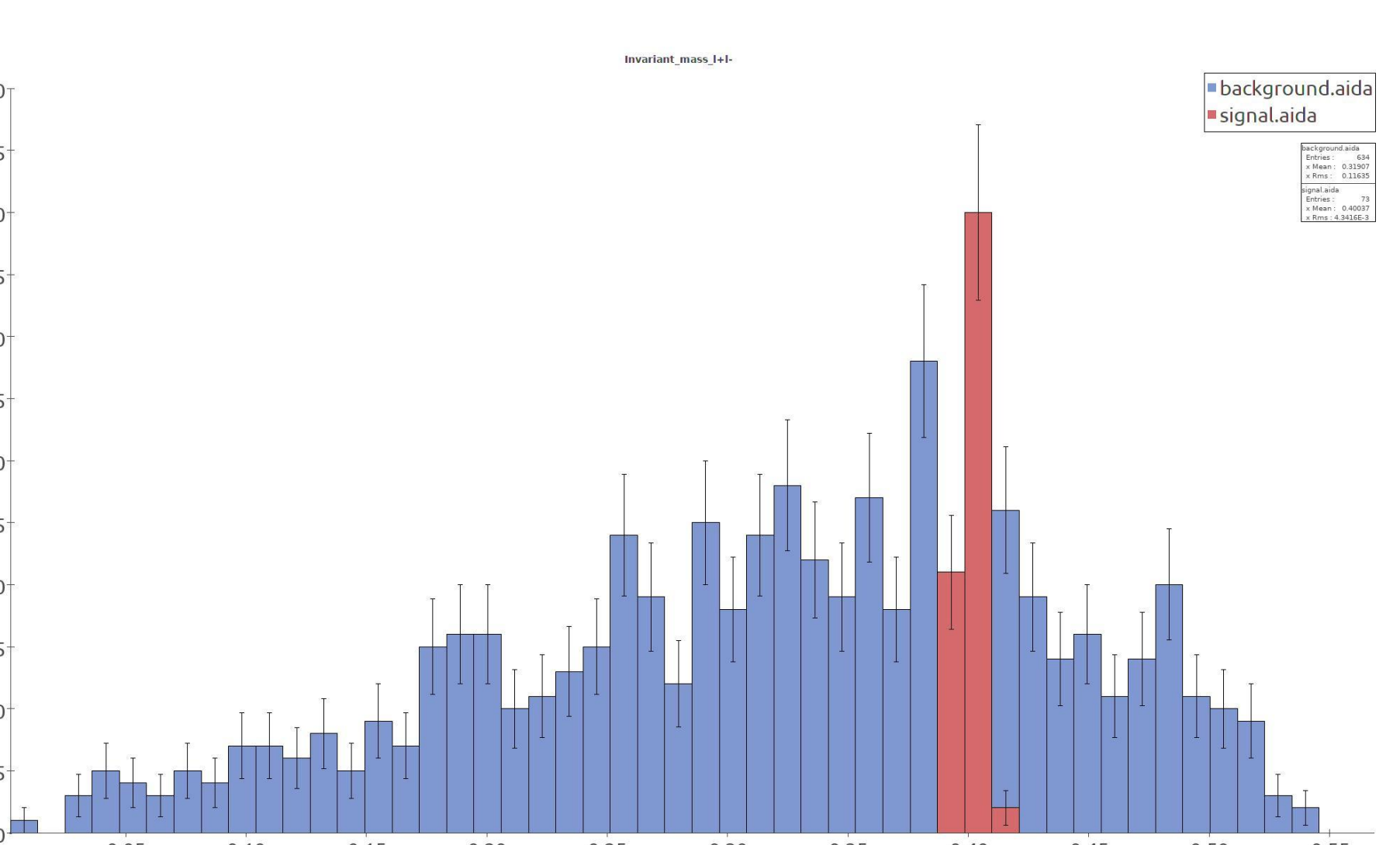

Figure 6: Background detections pictured in blue. Signal detections of $400 \mathrm{MeV}$ dark photon pictured in red

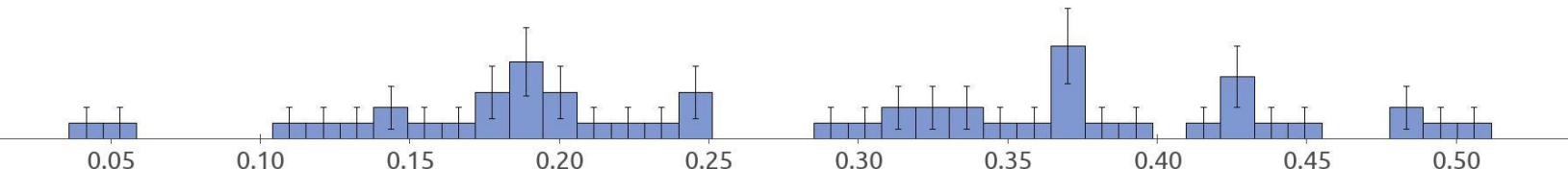

Figure 5: Background detections after cutting for secondary vertices

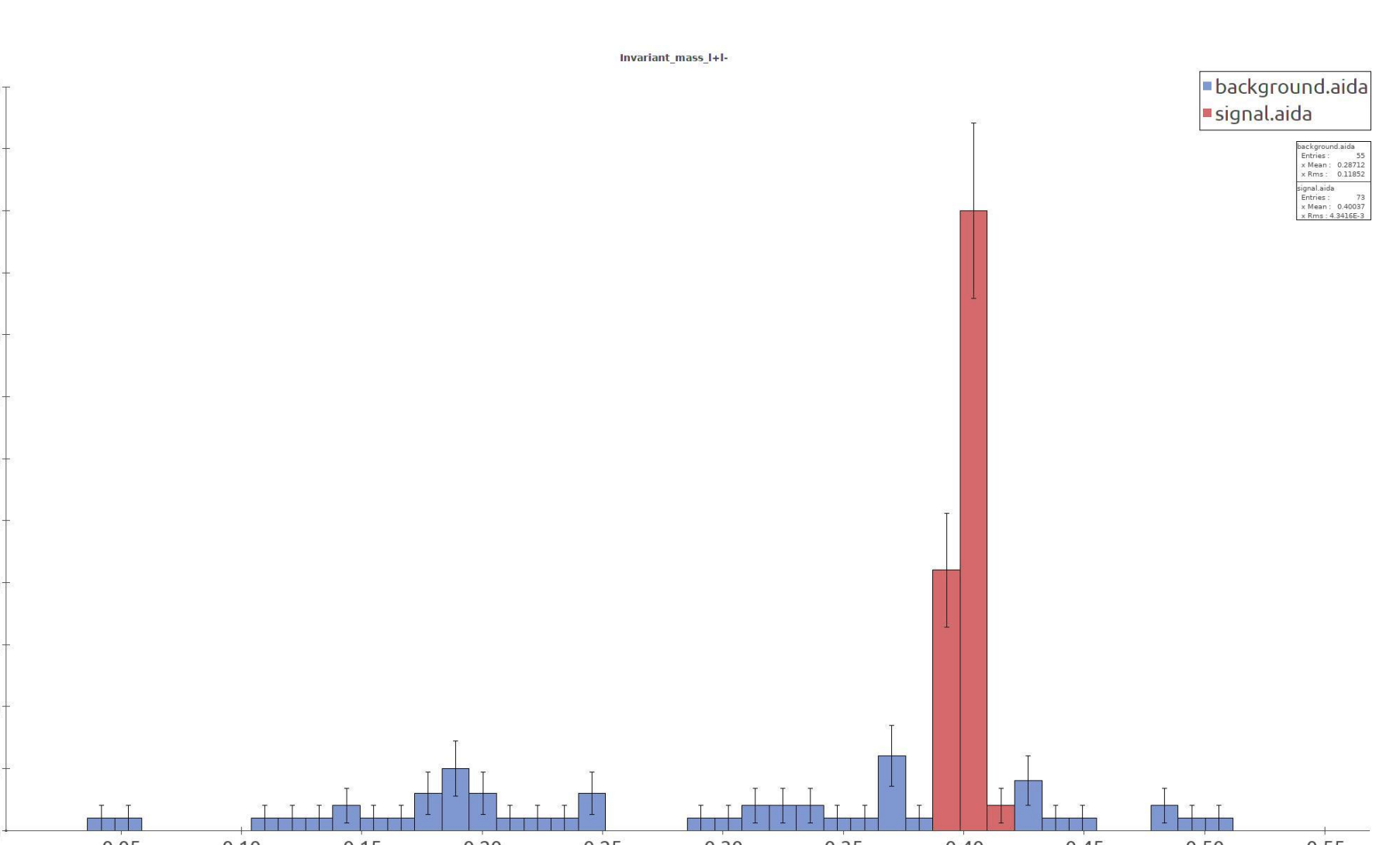

Figure 7: Background detections after cutting for secondary vertices pictured in blue. Signal detections of $400 \mathrm{MeV}$ dark photon pictured in red 